BULL. AUSTRAL. MATH. SOC.

$05 A 99,05499$

VOL. $26(1982), 343-354$.

\title{
ON A CLASS OF POLYNOMIALS ASSOCIATED WITH THE SUBGRAPHS OF A GRAPH AND ITS APPLICATION TO CHROMATIC AND DICHROMATIC POLYNOHIALS
}

\author{
E,J, FARRELL
}

Let $G$ be a graph. With every connected subgraph of $G$, let us associate a weight $w_{\alpha}$, and with every spanning subgraph (or cover) $C$ of $G$, a weight

$$
w(c)=\prod_{i=1}^{n} w_{\alpha_{i}},
$$

where $\alpha_{i}(i=1,2, \ldots, n)$ are the components of $C$. The subgraph polynomial of $G$ is

$$
\sum w(C)
$$

where the summation is taken over all the spanning subgraphs in $G$.

The basic properties of subgraph polynomials are given, expressions for the subgraph polynomials of multigraphs and pseudographs are derived, and the chromatic and dichromatic polynomials are shown to be special cases of the subgraph polynomial.

\section{Introduction}

The graphs considered here will be finite and undirected. Let $G$ be

Received 28 January 1982. 
such a graph. With every connected subgraph $\alpha$ of $G$, let us associate an indeterminante or weight $w_{\alpha}$. With every spanning subgraph (cover) $H$ of $G$, let us associate the weight

$$
w(H)=\prod_{\alpha} w_{\alpha}
$$

The subgraph polynomial of $G$ is

$$
\sum w(H)
$$

where the summation is taken over all covers $H$ in $G$.

In this paper we will initially assign weights to connected subgraphs according to the number of edges that they contain. Thus a connected subgraph with $n$ edges will be assigned a weight $w_{n}$. Hence the subgraph polynomial of $G$ will be a polynomial in the variables $w_{0}, w_{1}, w_{2}, \ldots$, and so on. If we denote the subgraph polynomial of $G$ by $S(G ; w)$, then we will have

$$
S(G ; w)=\sum A w_{0}^{i} o_{1}^{i_{1}{ }^{2} w_{2}{ }^{i}} \ldots w_{q}^{i} q,
$$

where $A$ is the number of spanning subgraphs of $G$, consisting of $i_{0}$ nodes, $i_{1}$ edges, $i_{2}$ trees with two edges, and so on, and $w=\left(w_{0}, w_{1}, w_{2}, \ldots\right)$ is a general weight vector. If we put $w_{i}=w$ for all $i$, then the resulting polynomial $S(G ; w)$ in the variable $w$, will be called the simple subgraph polynomial of $G$. In this case, the coefficient of $w^{k}$ will be the number of spanning subgraphs of $G$ with $k$ components. We note that the subgraph polynomial of a graph is a special kind of F-polynomial (see Farrell [1]).

We will give some of the basic properties of subgraph polynomials and deduce an algorithm for finding the polynomials. We will then derive expressions for the subgraph polynomials of pseudographs and multigraphs. We will show that the chromatic and dichromatic polynomials of a graph are special cases of its subgraph polynomial, when the weights are appropriately chosen. We refer the reader to Harary [2] for the basic definitions in graph theory. 


\section{The fundamental algorithm for subgraph polynomials}

Let $G$ be a graph. We can put the covers of $G$ into two classes;

(i) those containing a given edge $x y$, and

(ii) those not containing $x y$, where $x$ and $y$ are nodes in $V(G)$.

The covers which do not contain $x y$ will be covers of the graph $G^{\prime}$ obtained from $G$ by deleting $x y$. The covers containing $x y$ will be covers of the graph $G^{*}$ obtained from $G$ by incorporating $x y$; that is distinguishing $x y$ in some way and requiring it to belong to every cover of $G$ (for example, we can colour $x y$ ). Thus we have the fundamental theorem for subgraph polynomials.

THEOREM 1. $S(G ; W)=S\left(G^{\prime} ; w\right)+S\left(G^{*} ; w\right)$.

Since any subgraph of $G$ can be part of a cover, there are no restrictions on the edges which can be added to an incorporated subgraph. In addition to this, we are interested only in the number of edges in the incorporated subgraph (in this application). Therefore it is unnecessary to keep these subgraphs in their original forms, in a practical application of Theorem 1. We could instead "shrink" them to compound nodes, and keep an account of the number of edges in the compound nodes.

Identification of the nodes at the ends of the incorporated edge (in the sense described in Read [3]) will accomplish the shrinking process. Thus we can delete edges and identify nodes, just as we do for chromatic polynomials (see [3]). The only difference in this case is that we cannot ignore loops, for obvious reasons. Loops must be treated as ordinary edges. In order to keep an account of the number of edges in the compound nodes, we can assign numbers to these nodes, representing the number of edges in the incorporated subgraphs. Ordinary nodes can be given the number 0 . If two nodes with associated numbers $m$ and $n$ are identified, then the compound node formed by identifying them will receive an associated number $m+n+1$.

The fundamental algorithm for subgraph polynomials (or the reduction process, for brevity) consists of repeated applications of Theorem 1, until empty graphs result. Weights can then be assigned to these final empty graphs, and the polynomial written down. Of course, the incorporation 
process will vary according to the criteria used for assigning weights. Notice that if the simple subgraph polynomial is required, then the incorporation process could be simple node identification. This simplification is independent of the original criteria used for assigning weights .

The following is a useful corollary of the fundamental theorem. $T(G)$ denotes the number of connected spanning subgraphs of $G$.

COROLLARY 1.1. Let $G$ be a graph containing an edge $x y, G^{\prime}$ the graph obtained from $G$ by deleting $x y$ and $G^{*}$ the graph obtained from $G$ by identifying nodes $x$ and $y$. Then

$$
\tau(G)=\tau\left(G^{\prime}\right)+\tau\left(G^{*}\right) \text {. }
$$

Below is an illustration of the fundamental algorithm for subgraph polynomials.

The resulting polynomial is

$$
S(G ; w)=w_{0}^{4}+5 w_{0}^{2} w_{1}+8 w_{0} w_{2}+2 w_{0} w_{3}+2 w_{1}^{2}+8 w_{3}+5 w_{4}+w_{5} .
$$

\section{Basic properties of subgraph polynomials}

We can write the simple subgraph polynomial of $G$ as

$$
S(G ; w)=\sum_{k}^{p-1} a_{k} w^{p-k},
$$

where $p$ is the number of nodes in $G$ and $a_{k}$ is the number of subgraph covers with $p-k$ components. We will now prove the following lemma.

LEMMA 1 (The Cutnode Theorem). Let $G$ be a graph consisting of two blocks $D$ and $E$ with one common cutnode. Then

$$
S(G ; w)=w^{-1} S(D ; w) S(E ; w)
$$

Proof. Let $x$ be the node which is common to $D$ and $E$. Consider a cover with cardinality $k$. The components must be subgraphs belonging to $D$ only, to $E$ only and a subgraph of $D$ and $E$ "joined" together at node $x$. Thus two covers with cardinalities $m$ and $n$ in $D$ and $E$ respectively, yield a cover of $G$ with cardinality $m+n-1$.

If we give a weight $w$ to each component of each cover, then the 

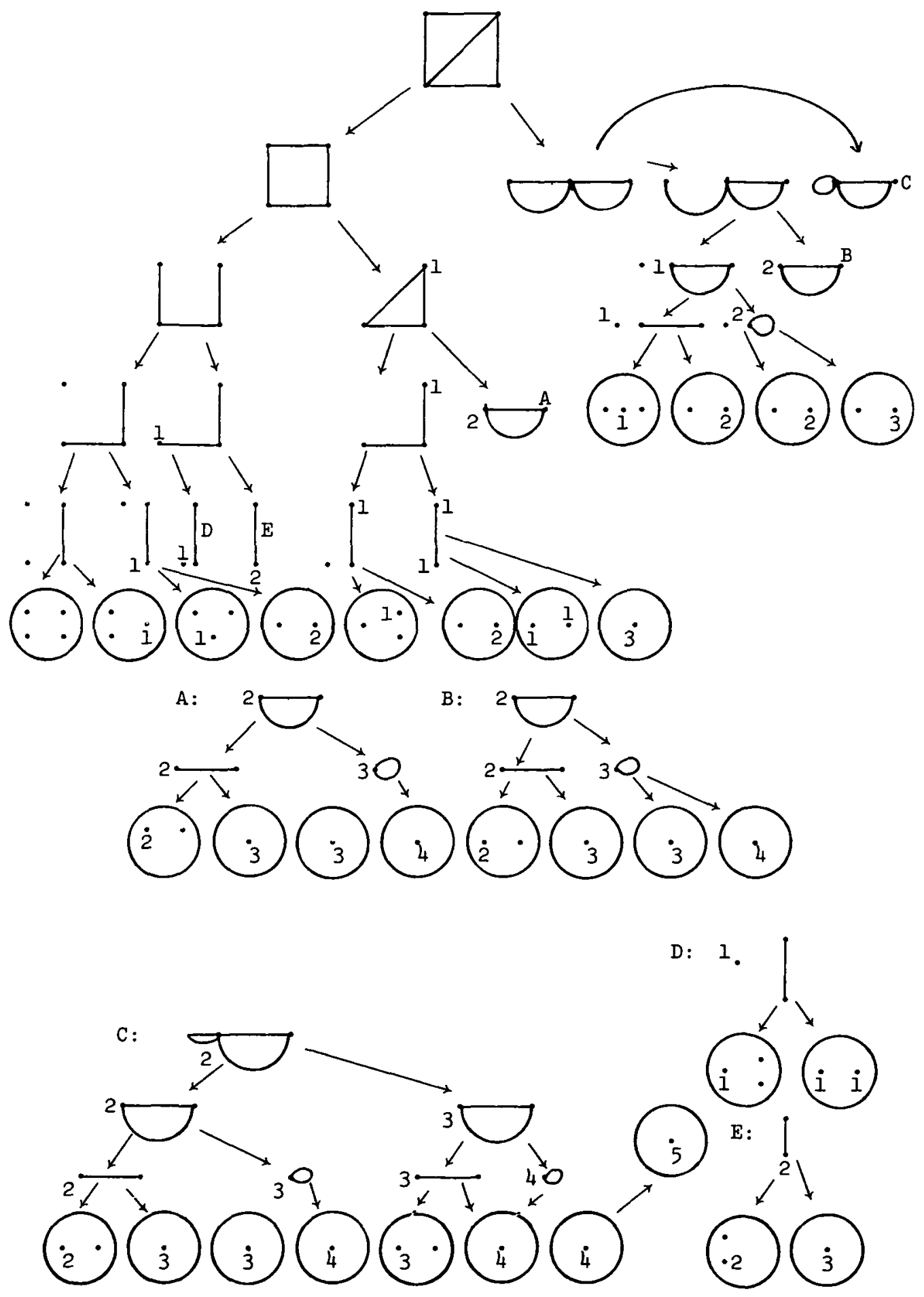
covers of $D$ and $E$ will receive weights of $w^{m}$ and $w^{n}$ respectively. However the resulting cover of $G$ will receive a weight of $w^{m+n-1}$. This will be true for all $m$ and $n$. Hence the result follows. //

This lemma can be easily extended to a graph containing several blocks.

THEOREM 2. Let $G$ be a graph consisting of $n$ blocks $B_{1}, B_{2}, \ldots, B_{n}$. Then

$$
S(G ; w)=w^{-(n-1)} \prod_{p=1}^{n} S\left(B_{r} ; w\right) .
$$

The following result can be easily proved.

THEOREM 3 (The Component Theorem). Let $G$ be a graph consisting of components $c_{1}, c_{2}, \ldots, c_{n}$. Then

$$
S(G ; w)=\prod_{i=1}^{n} S\left(C_{i} ; w\right) .
$$

The following theorem shows that there are no "gaps" in $S(G ; w)$.

THEOREM 4. The smallest exponent of $w$ in $S(G ; w)$ is the number of components in $G$, and all zarger powers of $w$, up to $w^{p}$, must occur in $S(G ; w)$ with nonzero coefficients.

Proof. Clearly $G$ is a cover of itself and no cover of $G$ can have less components than $G$ itself. Therefore the first part of the theorem follows.

The set of nodes of $G$ is a cover of $G$ and therefore $\omega^{p}$ will be the highest power of $w$ in $S(G ; w)$. Finally, any cover of $G$ with cardinality $r(r \neq p)$ can be transformed to a cover of $G$ with $r+1$ components by removing edges. Therefore the second part of the theorem follows. //

It is clear that the number of subgraphs in a graph $G$ with $q$ edges is $2^{q}$. Also, a spanning subgraph of $G$ is a cover with cardinality 1 . Thus $S(G ; W)$ has the following properties. 
PROPERTY 1. The sum of the coefficients of $S(G ; w)$ is $2^{q}$.

PROPERTY 2. The coefficient of $w_{p}$ in $S(G ; w)$ and the coefficient of $w$ in $S(G ; w)$ are equal to the number of spanning subgraphs in $G$.

$$
\text { PROPERTY 3. If } S(G ; w)=\sum_{k}^{p-1} a_{k} w^{p-k} \text { and } F(G ; w)=\sum_{k}^{p-1} b_{k} w^{p-k} \text { is }
$$

any other $F$-polynomial of $G$ (see [1]), then $a_{i} \geq b_{i}$ for all $i$.

\section{Subgraph polynomials of pseudographs and multigraphs}

We will obtain expressions for the subgraph polynomials of pseudographs and multigraphs. These results can be useful for deducing some well known properties of chromatic and dichromatic polynomials.

The following notations will be used. $G_{n}$ will represent a graph obtained from the graph $G$ by attaching $n$ loops to one of its nodes say node $x \cdot G_{n}^{*}[r]$ will be the graph obtained from $G_{n}$ by replacing node $x$ by a compound node "containing $r$ edges" (that is, a compound node representing a subgraph consisting of $r$ incorporated edges). Note that $G_{n}^{*}[r]$ will still have $n$ loops attached to the compound node $x$. For brevity, we will use $G$ itself for $S(G ; w)$ when it would lead to no confusion.

In the following theorem we give an expression for the subgraph polynomial of $G_{n}$.

THEOREM 5. $G_{n}=G+\left(1+G^{*}\right)^{n}-1$, where $\left(G^{*}\right)^{r} \equiv G^{*}[r]$.

Proof by induction on $n$. When $n=1$, the statement becomes

$$
G_{1}=G+G^{*}[1] \text {. }
$$

This is obviously true, as can be seen by applying the reduction process to $G_{1}$ by deleting the loop. Let us assume that the statement holds for $n=k$. Then

$$
G_{k}=G+\left(1+G^{*}\right)^{k}-1
$$


Let us apply the reduction process to $G_{k+1}$ by deleting one of the loops. This yields

$$
G_{k+1}=G_{k}+G_{k}^{*}[1]
$$

It follows from equation (1) with $G_{k}$ replaced by $G_{k}^{*}[1]$, that

$$
G_{k}^{*}[1]=G^{*}[1]+\left(1+G^{*}[1]\right)^{k}-1 \text {, }
$$

with

$$
\left(G^{*}[s]\right)^{p}=G^{*}[p+8]
$$

implying

$$
G_{k}^{*}[1]=G^{*}[1]+G^{*}[k+1]+\sum_{r=2}^{k}\left(\begin{array}{c}
k \\
r-1
\end{array}\right) G^{*}[r] .
$$

By substituting the expression given in (1) for $G_{k}$ and the expression for $G_{k}^{*}[1]$ given in (3), we obtain from (2),

$$
\begin{aligned}
G_{k+1} & =G+(k+1) G^{*}[1]+G^{*}(k+1)+\sum_{r=2}^{k}\left[\left(\begin{array}{l}
k \\
r
\end{array}\right)+\left(\begin{array}{c}
k \\
r-1
\end{array}\right)\right] G^{*}[r] \\
& =G+\sum_{r=1}^{k+1}\left(\begin{array}{c}
k+1 \\
r
\end{array}\right) G^{*}[r] \\
& =G+\left(1+G^{*}\right)^{k+1}-1 .
\end{aligned}
$$

Hence the statement holds for $n=k+1$, when it is assumed for $n=k$. Also it is true for $n=1$. By the Principle of Induction, it holds for all $n$. / /

Clearly if in $G_{n}$ node $x$ (the node to which the $n$ loops are attached) is a compound node containing an incorporated subgraph with edges, then Theorem 5 becomes

$$
G_{n}^{*}[i]=G^{*}[i]+\left(1+G^{*}[i]\right)^{n}-1
$$

Thus we get the following lemma.

LEMMA 2. $G_{n}^{*}[i]=\left(G^{*}\right)^{i}\left(1+G^{*}\right)^{n}$, with $\left(G^{*}\right)^{r} \equiv G^{*}[r]$. 
We will denote by $M_{n}$ the multigraph obtained from a graph $G$ by joining two non adjacent nodes $x$ and $y$ by $n$ edges. Applying the reduction process to $M_{n}$ by deleting one of the edges joining $x$ to $y$, we get

$$
M_{n}=M_{n-1}+G_{n-1}^{*}[1]
$$

By applying the reduction process in the same manner to the graph $M_{n-1}$, we get

$$
M_{n-1}=M_{n-2}+G_{n-2}^{*}[1]
$$

If the process is continued on the graphs $M_{n-2}, M_{n-3}$, and so on, we will get

$$
\begin{aligned}
& M_{n-2}=M_{n-3}+G_{n-3}^{*}[1] \\
& \vdots \quad \vdots \quad \vdots \\
& M_{1}=G+G_{0}^{*}[1] \text {. }
\end{aligned}
$$

Hence we get

$$
M_{n}=G+\sum_{k=1}^{n} G_{n-k}^{*}[1]
$$

By substituting for $G_{n-k}^{*}[1]$, using Lemma 2, we get

$$
M_{n}=G+\sum_{k=1}^{n} G^{*}\left(1+G^{*}\right)^{n-k}
$$

Thus we get the following theorem.

THEOREM 6. $M_{n}=G+G^{*}\left[\left(2+G^{*}\right)^{n}-\left(1+G^{*}\right)^{n}\right]$, with $\left(G^{*}\right)^{r} \equiv G^{*}[r]$.

\section{Application to chromatic polynomials}

We can write the subgraph polynomial of $G$ as

$$
S(G ; w)=\sum_{k=1}^{p} \sum_{(i)} a_{k}\left(i_{0}, i_{1}, i_{2}, \ldots, i_{q}\right) w_{0}^{i_{0}} w_{1}^{i_{1}} \ldots w_{q}^{i_{q}} \text {, }
$$


where $a_{k}\left(i_{0}, i_{1}, \ldots, i_{q}\right)$ is the number of covers with cardinality $k$ and containing $i_{0}$ isolated nodes, $i_{1}$ edges, and so on, and the second summation is taken over all solutions of $\sum_{r}^{q} i_{r}=k$.

Let us assign weights to the connected subgraphs as follows. If the subgraph contains $r$ edges it will be given a weight $(-1)^{r} \lambda$. Then equation (6) becomes

$$
S(G ; \lambda)=\sum_{k=1}^{p} \sum_{(i)} a_{k}\left(i_{0}, i_{1}, \ldots, i_{q}\right)(-1)^{n} \lambda^{k},
$$

where $n=\sum_{r=1} r i_{r}$. Since subgraphs with the same number of edges will receive the same weight, we can replace $a_{k}\left(i_{0}, i_{1}, \ldots, i_{q}\right)$ by $a_{k}(n)$ the number of covers with cardinality $k$ and containing $n$ edges. Thus equation (7) becomes

$$
\begin{aligned}
S(G ; \lambda) & =\sum_{k=1}^{p}\left[\sum_{n}^{q}(-1)^{n} a_{k}(n)\right] \lambda^{k} \\
& =\sum_{k=1}^{p} a_{k} \lambda^{k},
\end{aligned}
$$

where

$$
a_{k}=\sum_{n}^{q}(-1)^{n} a_{k}(n)
$$

Whitney [4] has shown that $d_{k}$ is the coefficient of the kth power of the variable in the chromatic polynomial of the graph. (A proof is also given in [3].) This implies the following theorem.

THEOREM 7. The polynomial obtained from $S(G ; w)$ by putting $w_{n}=(-1)^{r} \lambda$, is the chromatic polynomial of $G$.

\section{Application to dichromatic polynomials}

Let us assign weights to the subgraph covers of a graph $G$, as follows. If a component contains $n$ nodes and $e$ edges, then it will be 
given a weight $w_{n, e}$. Then the general subgraph polynomial of $G$ weighted by both nodes and edges is

$$
S(G ; w)=\sum \prod_{i, j} w_{i, j}^{n},
$$

where the summation is taken over all subgraph covers of $G$.

Let us now assign special weights to components by putting

$$
w_{n, e}=x y^{e-n+1}
$$

Then equation ( 8 ) becomes

$$
D(G ;(x, y))=\sum x^{\delta_{k} \gamma_{k}},
$$

where $\delta_{k}$ is the number of components in the $k$ th cover and $\gamma_{n}=q_{k}-p_{k}+\delta_{k}$, where $p_{k}$ and $q_{k}$ are the number of nodes and edges respectively in the $k$ th cover of $G$. The summation is taken over all the subgraph covers of $G$.

Clearly $\gamma_{k}$ and $p_{k}-\delta_{k}$ are respectively the cycle rank and coboundary rank of the cover (see Tutte [4]). It follows therefore, that $D(G ;(x, y))$ is precisely the dichromatic polynomial of $G$, defined in [4]. Hence we have the following theorem.

THEOREM 8. The polynomial obtained from $S(G ; w)$ (equation (8)) by putting $w_{n, e}=x y^{e-n+1}$, is the dichromatic polynomial of $G$.

\section{Discussion}

We have introduced the subgraph polynomial and have shown that it is a generalization of both the chromatic and dichromatic polynomials. It might be interesting to investigate the extent to which the subgraph polynomial of a graph characterizes a graph. We have found that it is difficult to obtain two different graphs with the same subgraph polynomial even when the weights simply depend on the number of edges in the covers. Perhaps these polynomials might shed some light on the isomorphism problem. It might also be interesting to look at subgraph polynomials of graphs in which 
certain components must occur in all subgraph covers.

\section{References}

[1] E.J. Farrell, "On a general class of graph polynomials", J. Combin. Theory Ser. B 26 (1979), 111-122.

[2] Frank Harary, Graph theory (Addison-Wesley, Reading, Massachusetts; Menlo Park, California; London; 1969).

[3] Ronald C. Read, "An introduction to chromatic polynomials", J. Combin. Theory 4 (1968), 52-71.

[4] W.T. Tutte, "On dichromatic polynomials", J. Combin. Theory 2 (1967), $301-320$.

[5] Hassler Whitney, "A logical expansion in mathematics", Bulz. Amer. Math. Soc. 38 (1932), 572-579.

Department of Mathematics, University of West Indies,

St Augustine, Trinidad, West Indies. 